\title{
Youth Mentoring: New Areas for Research
}

\author{
Melissa F. Peskin
}

Published online: 3 December 2011

(C) Springer Science+Business Media, LLC 2011

This issue includes a diverse array of articles on parenting and obesity prevention, the cost benefits of financial incentives for participation in parenting programs, diabetes and disability prevention, communication practices between college students and their physicians, and youth mentoring. Although each contributes greatly to prevention science, this editorial will focus on the two youth mentoring articles, which I believe cover important areas for mentoring research: cultural competence of mentoring programs and effects of mentoring for mentors.

Years of research have revealed the positive benefits of mentoring programs in increasing academic outcomes and reducing risk behaviors for youth (Sipe, 2002). However, both articles in this issue highlight the need for additional rigorous evaluation in understanding the effects of mentoring programs for both mentees and mentors. In the first article, Farruggia and colleages provide a systematic review of mentoring programs in New Zealand. These authors have taken a critical first step in evaluating mentoring programs, a bourgeoning new research area in New Zealand, taking into account the importance of cultural relevancy and research methodology. In their review, many

M. F. Peskin $(\bowtie)$

Health Promotion and Behavioral Sciences,

School of Public Health, The University of Texas,

Houston, TX, USA

e-mail: melissa.f.peskin@uth.tmc.edu programs, specifically those that targeted Maori youth in New Zealand, lacked significant cultural elements, such as inclusion of families, in their programming. Although they present an unexpected finding - namely that as the cultural appropriateness of programs increases, program effectiveness decreases - their conclusions still address the importance of integrating culture in mentoring programs. The authors state that one of the reasons for this finding could be the lack of culturally appropriate research methods used to evaluate some programs in their review.

In the second article, Faith and colleagues describe an interesting study in which they examine the effects of mentoring on mentors' self-efficacy for mentoring, attitudes towards future parenting, personality traits, and avoidance/ambivalence attachment tendencies. Their contention is that though mentoring has positive effects for the mentored youth, more studies are needed to examine the effect of mentoring on the actual mentors, particularly those who work with highly aggressive youth. The study findings suggest some potential negative effects for mentors in the areas of mentoring self-efficacy, openness, conscientiousness, extraversion, and agreeableness. On a positive note, mentors who perceived a more supportive relationship with their mentees were more likely to exhibit some positive effects in these outcomes. Although enthusiasm for the findings might be somewhat tempered by the use of their one-group design, the authors shed light on an issue that may be too little examined in the mentoring literature. 
Together, both articles provide critical insights into future research that needs to be conducted on youth mentoring. Further, both share a commonality in that they point to the need for additional rigorous evaluation research on mentoring programs, in terms of understanding their impact in countries outside of the United States as well as on the mentors themselves. This is a central consideration as we continue to implement and disseminate mentoring programs to youth.

\section{Reference}

Sipe, C. L. (2002). Mentoring programs for adolescents: A research summary. Journal of Adolescent Health, 31(6 Suppl), 251-260. 